\title{
PK-R Allosteric Activator AG-348
}

National Cancer Institute

\section{Source}

National Cancer Institute. PK-R Allosteric Activator AG-348. NCI Thesaurus. Code C157039.

An orally available, small-molecule, allosteric activator of the red cell isoform of pyruvate kinase (PK-R), with potential to improve hemolytic anemia in patients with pyruvate kinase deficiency (PKD). Upon oral administration, AG-348 binds to and activates PK-R, thereby enhancing glycolytic pathway activity, improving adenosine triphosphate (ATP) levels and reducing 2,3-diphosphoglycerate (2,3-DPG) levels. Mutations in PK-R cause deficiency in PK-R which prevents adequate red blood cell (RBC) glycolysis, leading to a buildup of the upstream glycolytic intermediate 2,3-DPG and deficiency in the PK-R product ATP. 